I. Satake

Nagoya Math. J

Vol. 62 (1976) 1-12

\title{
ON CLASSIFICATION OF QUASI-SYMMETRIC DOMAINS
}

\section{Dedicated to the memory of Taira Honda}

\section{SATAKE}

The notion of "Siegel domains" was introduced by Pjateckii-Šapiro [8]. It was then shown that every homogeneous bounded domain is holomorphically equivalent to a Siegel domain (of the second kind) determined uniquely up to an affine isomorphism ([15], cf. also [2], [4], [9b]). In a recent note [10b], I have shown that among (homogeneous) Siegel domains the symmetric domains can be characterized by three conditions (i), (ii), (iii) on the data $(U, V, \Omega, F)$ defining the Siegel domain (see Theorem in $\S 2$ of this paper $)^{1)}$. The class of homogeneous Siegel domains satisfying partial conditions (i), (ii), which we propose to call "quasi-symmetric", seems to be of some interest, since for instance the fibers appearing in the expressions of symmetric domains as Siegel domains of the third kind fall in this class ([10b], [16]). Recently, using a method of $S$-algebras ([11a, b]), Takeuchi [11c] gave a complete classification of quasi-symmetric domains, which naturally implies a new classification of symmetric domains ${ }^{2)}$. The purpose of the present note is to show that this classification can also be obtained immediately from my previous result on linear imbeddings of self-dual cones ([10a]).

Our method is based on the following two observations:

(I) There are natural equivalences between the three categories of (punctured) self-dual cones, the corresponding reductive Lie algebras (with fixed Cartan involutions), and formally real Jordan algebras (§1).

(II) There is a natural bijection between the set of isomorphism classes of quasi-symmetric Siegel domains and that of equivalence classes of the pairs formed of a self-dual cone and a (linear) "representation" of it ( $\S 3$, Proposition 2).

Received October 7, 1975.

1) A similar result was also obtained independently by J. Dorfmeister.

2) Several results toward the classification of Siegel domains satisfying only the condition (i) have been obtained by Takeuchi [11b], Tsuji [13] and others. 
It follows that the classification of quasi-symmetric Siegel domains with a fixed self-dual cone $\Omega$ amounts to the determination of all "representations" of $\Omega$. This is precisely what was done in [10a] in terms of the corresponding Lie algebras $\mathfrak{g}(\Omega)$. For example, if $\Omega$ is the exceptional irreducible self-dual cone $\mathscr{P}_{3}(\boldsymbol{O})$, then the only possible representation is the trivial one ${ }^{3}$, which corresponds to the symmetric tube domain $U+i \Omega$, i.e., the exceptional irreducible symmetric domain of type $\left(E_{7}\right)$. An open problem in this direction is to give an explicit description of morphisms (e.g. strongly equivariant holomorphic maps) between quasisymmetric domains in terms of the corresponding pairs mentioned in (II). ${ }^{4)}$ It would also be interesting to find an analytic or differential geometric characterization of quasi-symmetric domains.

\section{§1. Self-dual cones}

Let $U$ be a (finite-dimensional) real vector space. By a cone in $U$ we always mean a non-empty open convex cone in $U$ with vertex at the origin and not containing any straight line. A cone $\Omega$ in $U$ is called "homogeneous" if the linear automorphism group

$$
G(\Omega)=\{A \in G L(U) \mid A \Omega=\Omega\}
$$

acts transitively on $\Omega$. We fix once and for all a positive-definite inner product $\langle>$ on $U$ and set

$$
\Omega^{*}=\left\{u \in U \mid\left\langle u, u^{\prime}\right\rangle>0 \quad \text { for all } u^{\prime} \in \bar{\Omega}-\{0\}\right\},
$$

where $\bar{\Omega}$ is the topological closure of $\Omega . \quad \Omega$ is called "self-dual" if $\Omega$ is homogeneous and $\Omega^{*}=\Omega$, or equivalently, ${ }^{t} G(\Omega)=G(\Omega), t$ denoting the adjoint with respect to \langle\rangle .

Let $\Omega$ be a self-dual cone in $U$. Then it is known ([14]) that $G(\Omega)$ is an open subgroup of a reductive real algebraic group and the isotropy subgroup $K_{a}$ of $G(\Omega)$ at any point $a \in \Omega$ is a maximal compact subgroup. It follows that there exists an element $e \in \Omega$ such that

$$
K_{e}=\left\{\left.A \in G(\Omega)\right|^{t} A=A^{-1}\right\} \text {. }
$$

Let $g(\Omega)$ be the (linear) Lie algebra of $G(\Omega)(\subset g l(U))$. Then the Cartan involution of $g(\Omega)$ at $e$ is given by

3) This is essentially a theorem of Albert (Ann. of Math. 35 (1934)).

4) For the subcategory of symmetric tube domains, this is one of the questions raised in [10a], which can easily be answered by using the description of automorphism groups of symmetric tube domains in terms of Jordan algebras ([5a, c], [9b], [10b]). 


$$
\theta_{e}: A \longmapsto-{ }^{t} A \text {, }
$$

and we have the Cartan decomposition:

$$
\mathfrak{g}(\Omega)=\mathfrak{f}_{e}+\mathfrak{p}_{e},
$$

where $\mathfrak{f}_{e}=\left\{\left.A \in g(\Omega)\right|^{t} A=-A(\Leftrightarrow A e=0)\right\}$ is the Lie algebra of $K_{e}$. The "reference point" $e$ may be chosen arbitrarily by the homogeneity of $\Omega$, but will be fixed throughout the paper.

From the above, it is clear that, for every $u \in U$, there exists a unique element $T_{u}$ in $\mathfrak{p}_{e}$ such that

$$
T_{u}(e)=u
$$

in particular, $T_{e}=1_{U}$ (the identity transformation of $U$ ). The correspondence $u \mapsto T_{u}$ gives a linear isomorphism $U \cong \mathfrak{p}_{\ell}$. It is known ([1], [5a], [14]) that, if we define a product $\circ$ in $U$ by

$$
u_{1} \circ u_{2}=T_{u_{1}}\left(u_{2}\right) \quad\left(u_{1}, u_{2} \in U\right),
$$

then $U$ becomes a Jordan algebra with the unit element $e$, which is "formally real" (or "compact") in the sense that $u_{1}^{2}+u_{2}^{2}=0$ implies $u_{1}$ $=u_{2}=0$, or equivalently, that $\operatorname{tr}\left(T_{u ః}\right)$ is a positive-definite quadratic form on $U$. Moreover, $\Omega$ coincides with the interior of $\left\{u^{2} \mid u \in U\right\}$. Conversely, all formally real Jordan algebras are obtained in this manner from self-dual cones.

Now let $\Omega^{\prime}$ be another self-dual cone in a real vector space $U^{\prime}$ with a reference point $e^{\prime}$. We use a similar notation as above with primes to denote the objects relative to $\left(\Omega^{\prime}, e^{\prime}\right)$; e.g., $T_{u^{\prime}}^{\prime}\left(u^{\prime} \in U^{\prime}\right)$ denotes the unique element in $\mathfrak{p}_{e^{\prime}}^{\prime}$ such that $T_{u^{\prime}}^{\prime}\left(e^{\prime}\right)=u^{\prime}$. The following Lemma is fundamental.

Lemma. Let $\varphi: U \rightarrow U^{\prime}$ be a linear map with $\varphi(e)=e^{\prime}$. Then $\varphi$ is a Jordan algebra homomorphism, if and only if there exists a Lie algebra homomorphism $\rho: \mathfrak{g}(\Omega) \rightarrow \mathrm{g}^{\prime}\left(\Omega^{\prime}\right)$ satisfying the following conditions:

$$
\begin{gathered}
\varphi(A u)=\rho(A) \varphi(u) \quad \text { for all } A \in \mathfrak{g}(\Omega), u \in U, \\
\rho \cdot \theta_{e}=\theta_{e^{\prime}}^{\prime} \cdot \rho .
\end{gathered}
$$

When this is the case, $\rho$ and $\varphi$ determine each other uniquely by the relation

$$
\rho\left(T_{u}\right)=T_{\varphi(u)}^{\prime}
$$


Moreover, one has $\varphi(\Omega) \subset \Omega^{\prime}$.

Proof. First, suppose there exists a Lie algebra homomorphism $\rho$ satisfying the conditions (7), (8). Then from (8) one has $\rho\left(\mathfrak{f}_{e}\right) \subset \mathfrak{f}_{e^{\prime}}^{\prime}$, $\rho\left(\mathfrak{p}_{e}\right) \subset \mathfrak{p}_{e^{\prime}}^{\prime}$. Hence, by (7) and the uniqueness of $T_{u^{\prime}}^{\prime}$, one obtains the relation (9). It follows that

$$
\varphi\left(u_{1} \circ u_{2}\right)=\varphi\left(T_{u_{1}}\left(u_{2}\right)\right)=\rho\left(T_{u_{1}}\right) \varphi\left(u_{2}\right)=T_{\varphi\left(u_{1}\right)}^{\prime} \varphi\left(u_{2}\right)=\varphi\left(u_{1}\right) \circ \varphi\left(u_{2}\right),
$$

i.e., $\varphi$ is a Jordan algebra homomorphism. Conversely, suppose $\varphi$ is a Jordan algebra homomorphism and define a linear map $\rho: \mathfrak{p}_{e} \rightarrow \mathfrak{p}_{e^{\prime}}$ by (9). Then, by the following well-known identity in a Jordan algebra:

$$
\left[T_{u_{1}},\left[T_{u_{2}}, T_{u_{3}}\right]\right]=T_{\left(u_{1} \circ u_{2}\right) \circ u_{3}}-T_{\left(u_{1} \circ u_{3}\right) u_{2}} \quad\left(u_{1}, u_{2}, u_{3} \in U\right),
$$

one has

$$
\rho\left(\left[T_{u_{1}},\left[T_{u_{2}}, T_{u_{3}}\right]\right]\right)=\left[\rho\left(T_{u_{1}}\right),\left[\rho\left(T_{u_{2}}\right), \rho\left(T_{u_{8}}\right)\right]\right] .
$$

Since $g(\Omega)$ is reductive and generated by $\mathfrak{p}_{e}$, it follows that $\rho$ can uniquely be extended to a Lie algebra homomorphism $g(\Omega) \rightarrow g\left(\Omega^{\prime}\right)$. It is then clear that the conditions (7), (8) are satisfied. Finally, from (7) one obtains $\varphi(\Omega)=\varphi\left(G(\Omega)^{\circ} e\right) \subset G\left(\Omega^{\prime}\right)^{\circ} e^{\prime}=\Omega^{\prime}$, where the superscript ${ }^{\circ}$ denotes the identity connected component of a topological group, q.e.d.

A map of a self-dual cone $\Omega$ into another self-dual cone $\Omega^{\prime}$ is called an equivariant (resp. strongly equivariant) linear map of $(\Omega, e)$ into $\left(\Omega^{\prime}, e^{\prime}\right)$, if it is the restriction of a linear map $\varphi: U \rightarrow U^{\prime}$ with $\varphi(e)=e^{\prime}$ such that there exists a Lie algebra homomorphism $\rho$ satisfying (7) (resp. (7),(8)). ${ }^{5)}$ The above Lemma implies that the following three categories are equivalent:

(a) the category of (punctured) self-dual cones $(\Omega, e)$, morphisms being strongly equivariant linear maps;

(b) the category of the corresponding reductive Lie algebras $g(\Omega)$ with fixed Cartan involutions $\theta_{e}$, morphisms being Lie algebra homomorphisms satisfying (7), (8) (with $\varphi$ defined by (9));

(c) the category of formally real Jordan algebras $U$, morphisms being (unital) Jordan algebra homomorphisms.

Thus, in particular, the classification of self-dual cones (up to linear isomorphisms) is equivalent to that of formally real Jordan algebras ([1], [14]). In order to fix the notation which will be used in the next section,

5) It seems likely that any equivariant linear map is necessarily strongly equivariant. This is true at least for $\Omega^{\prime}=\mathscr{P}_{m}(C)$ (see the proof of Proposition 1). 
we give here a description of the classical cones over the complex field $C$. Let $V$ be a (finite-dimensional) vector space over $C$ and $f$ a positivedefinite hermitian form on $V$. We denote by $\mathscr{H}(V, f)$ the real vector space of all "hermitian" (i.e. self-adjoint) transformations of $V$ with respect to $f$, and by $\mathscr{P}(V, f)$ the subset of $\mathscr{H}(V, f)$ formed of all positive-definite transformations. Then $\Omega=\mathscr{P}(V, f)$ is a self-dual cone in $U=\mathscr{H}(V, f)$ with respect to the inner product $\left\langle H_{1}, H_{2}\right\rangle=\operatorname{tr}\left(H_{1} H_{2}\right)$. The full endomorphism algebra $\mathfrak{g} \mathfrak{l}(V)$ acts on $\mathscr{H}(V, f)$ by

$$
(B, H) \longmapsto B H+H B^{*} \quad(B \in \operatorname{gl}(V), H \in \mathscr{H}(V, f)),
$$

* denoting the adjoint with respect to $f$. This action gives rise to a surjective homomorphism $\mathrm{gl}(V) \rightarrow \mathrm{g}(\Omega)$ with kernel $\left\{\sqrt{-1} \lambda \mathbf{1}_{V} \mid \lambda \in R\right\}$. Hence, if we set

$$
\mathfrak{g l}^{0}(V)=\{B \in \mathfrak{g l}(V) \mid \operatorname{tr} B \in R\},
$$

then $\mathfrak{g}(\Omega)$ can naturally be identified with $\mathfrak{g l}^{0}(V)$. The Jordan product in $\mathscr{H}(V, f)$ with the unit element $e=1_{V}$ is given by

$$
H_{1} \circ H_{2}=\frac{1}{2}\left(H_{1} H_{2}+H_{2} H_{1}\right) \quad\left(H_{1}, H_{2} \in \mathscr{H}(V, f)\right) .
$$

When $V=C^{m}$ and $f$ is the standard hermitian form on $C^{m}$, we write $\mathscr{H}_{m}(\boldsymbol{C})$ and $\mathscr{P}_{m}(\boldsymbol{C})$ for $\mathscr{H}(V, f)$ and $\mathscr{P}(V, f)$. In [10a] we have determined all possible equivariant linear maps of an arbitrary self-dual cone $(\Omega, e)$ into $\left(\mathscr{P}_{m}(C), 1_{m}\right)$.

\section{§2. Siegel domains}

To define a Siegel domain (of the second kind), we need the following data:

$U=$ a (finite-dimensional) real vector space,

$V=$ a (finite-dimensional) complex vector space,

$\Omega=$ an (open convex) cone in $U$,

$F=$ an $\Omega$-hermitian form on $V$,

where an " $\Omega$-hermitian form" $F$ is a hermitian sesquilinear map $F$ : $V \times V \rightarrow U_{C}=U \otimes_{R} C$ (which we assume to be $C$-linear in the second variable) satisfying the condition

$$
F(v, v) \in \bar{\Omega}-\{0\} \quad \text { for all } v \in V, v \neq 0 \text {. }
$$

A Siegel domain $\mathscr{D}=\mathscr{D}(U, V, \Omega, F)$ is then defined by 


$$
\mathscr{D}=\left\{(u, v) \in U_{c} \times V \mid \operatorname{Im} u-F(v, v) \in \Omega\right\} .
$$

We denote by $G=\mathrm{Hol}(\mathscr{D})$ the Lie group of all holomorphic automorphisms of $\mathscr{D}$ and by $\mathfrak{g}=\mathfrak{g}(\mathscr{D})$ its Lie algebra. It is known ([4]) that one has a natural gradation:

$$
\mathfrak{g}=\mathfrak{g}_{-1}+\mathfrak{g}_{-\frac{1}{2}}+\mathfrak{g}_{0}+\mathfrak{g}_{\frac{1}{2}}+\mathfrak{g}_{1}
$$

such that the non-positive part $\mathfrak{g}_{-}=\sum_{\nu \leq 0} \mathfrak{g}_{\nu}$ is the Lie algebra of the affine automorphism group Aff $(\mathscr{D})$. More precisely, $\mathfrak{g}_{-1}, \mathfrak{g}_{-\frac{1}{2}}$, and $\mathfrak{g}_{0}$ can naturally be identified with $U, V$, and the following subalgebra of $g(\Omega)$ $\oplus \mathfrak{g l}(V)$ :

$$
\{(A, B) \mid A \in \mathfrak{g}(\Omega), B \in \mathfrak{g l}(V), B \text { associated to } A\},
$$

respectively, where " $B$ is associated to $A$ " (with respect to $F$ ) means that the relation

$$
A F\left(v_{1}, v_{2}\right)=F\left(B v_{1}, v_{2}\right)+F\left(v_{1}, B v_{2}\right)
$$

holds for all $v_{1}, v_{2} \in V$. If the Siegel domain $\mathscr{D}$ is homogeneous, i.e.,

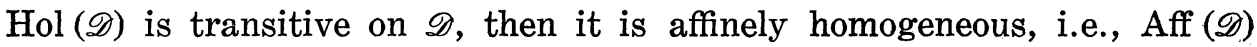
is transitive on $\mathscr{D}$, and hence the cone $\Omega$ is also homogeneous. When $\mathscr{D}$ is homogeneous, the positive part of $\mathrm{g}$ can be described explicitly in terms of $g_{-}(\mathrm{cf} .[6],[10 \mathrm{~b}],[12])^{6}$.

Now, for $u \in U$, we set

$$
F_{u}\left(v_{1}, v_{2}\right)=\left\langle u, F\left(v_{1}, v_{2}\right)\right\rangle,
$$

where \langle\rangle is the (symmetric) $C$-bilinear extension to $U_{C} \times U_{C}$ of the fixed inner product on $U$. Then $F_{u}(u \in U)$ is a hermitian form on $V$ in the ordinary sense, and is positive-definite if $u \in \Omega^{*}$. In particular, when $\Omega$ is self-dual, $f=F_{e}$ is a positive-definite hermitian form. In that case, we define $R_{u} \in \mathfrak{g l}(V)$ by

$$
F_{u}\left(v_{1}, v_{2}\right)=2 f\left(v_{1}, R_{u} v_{2}\right) .
$$

Then $R_{u} \in \mathscr{H}(V, f)$, and $R_{u} \in \mathscr{P}(V, f)$ if $u \in \Omega$; in particular, $R_{e}=\frac{1}{2} 1_{V}$. In this notation, the relation (15) can be rewritten as

$$
R_{t_{\Delta u}}=B^{*} R_{u}+R_{u} B \quad(u \in U) .
$$

We denote by $R$ the linear map $U \rightarrow \mathscr{H}(V, f)$ given by $u \mapsto R_{u}$. (We also

6) For the treatment of non-homogeneous case, see [3], [7], [9b]. 
use the same notation $R$ to denote its $C$-linear extension $U_{C} \rightarrow \mathfrak{g r}(V)$.)

As is well-known, $\mathscr{D}$ is symmetric if and only if $\mathscr{D}$ is homogeneous and $g(\mathscr{D})$ is semi-simple. In that case, $g_{0}$ (being the centralizer of a semi-simple element $\left.\left(1_{U}, \frac{1}{2} 1_{V}\right)\right)$ is reductive, and hence $\Omega$ is self-dual. In [10b], we gave the following characterization of symmetric domains.

THEOREM. A Siegel domain $\mathscr{D}=\mathscr{D}(U, V, \Omega, F)$ is symmetric if and only if the following three conditions are satisfied (for a suitable $\langle>$ ):

(i) $\Omega$ is self-dual.

(ii) For every $u \in U, R_{u}$ is associated to $T_{u}$.

(iii) The following relation is satisfied for all $v_{1}, v_{2}, v_{3} \in V$

$$
F\left(v_{1}, R\left(F\left(v_{2}, v_{3}\right)\right) v_{3}\right)=F\left(R\left(F\left(v_{3}, v_{1}\right)\right) v_{2}, v_{3}\right) .
$$

When $\mathscr{D}$ is symmetric, the Cartan involution $\theta$ of $g$ at $(\sqrt{-1} e, 0)$ reverses the gradation: $\theta\left(\mathfrak{g}_{\nu}\right)=\mathfrak{g}_{-\nu}$, and on $\mathfrak{g}_{0}$ one has

$$
\theta:(A, B) \longmapsto\left(-{ }^{t} A,-B^{*}\right) \text {. }
$$

Moreover, explicit expressions can be given for $\theta(u)$ and $\theta(v)\left(u \in U=\mathfrak{g}_{-1}\right.$, $\left.v \in V=\mathfrak{g}_{-\frac{1}{2}}\right)([10 \mathrm{~b}])$.

\section{§3. Quasi-symmetric Siegel domains}

First we prove the following proposition concerning the second condition (ii).

Proposition 1. Under the condition (i), the following four conditions are equivalent:

$\left(\mathrm{ii}_{1}\right)(=(\mathrm{ii}))$ For every $u \in U, R_{u}$ is associated to $T_{u}$.

(ii $\left.{ }_{2}\right)$ The map $2 R: u \mapsto 2 R_{u}$ is a Jordan algebra homomorphism of $U$ into $\mathscr{H}(V, f)$.

$\left(\mathrm{ii}_{3}\right)$ There exists a (unique) Lie algebra homomorphism $\beta: g(\Omega) \rightarrow$ $\mathfrak{g l}^{0}(V)$ such that

(20) $\beta(A)$ is associated to $A$, i.e., $R_{t_{A u}}=\beta(A)^{*} R_{u}+R_{u} \beta(A)$ for all $u \in U$,

$$
\beta\left({ }^{t} A\right)=\beta(A)^{*} \quad(A \in \mathfrak{g}(\Omega)) .
$$

(ii $\left.{ }_{4}\right)$ The projection map $(A, B) \mapsto A$ of $\mathrm{g}_{0}$ into $\mathrm{g}(\Omega)$ is surjective.

Proof. In view of $(6),(11)$, and $\left(15^{\prime}\right),\left(\mathrm{ii}_{1}\right)$ is equivalent to $\left(\mathrm{ii}_{2}\right)$. 
Applying Lemma to $U^{\prime}=\mathscr{H}(V, f), \Omega^{\prime}=\mathscr{P}(V, f)$, and $e^{\prime}=1_{V}$, we see that $\left(\mathrm{ii}_{2}\right)$ is equivalent to $\left(\mathrm{ii}_{3}\right)$. Note that, since $\mathrm{g}(\mathscr{P}(V, f))$ is identified with $\mathrm{gl}^{0}(V)$ by the action (10), the conditions (20), (21) in (ii $\left.{ }_{3}\right)$ coincide with (7), (8) with $\varphi=2 R$. It should also be noted that the image of a homomorphism $\beta: \mathfrak{g}(\Omega) \rightarrow \mathfrak{g l}(V)$ satisfying (21) is necessarily contained in $\mathfrak{g l}^{l^{0}}(V)$. Indeed, since $\mathfrak{f}_{e}$ is contained in the semi-simple part of $g(\Omega)$, one has $\beta\left(\mathfrak{f}_{e}\right) \subset \mathfrak{g l}(V)$. Hence, for $A \in \mathfrak{g}(\Omega)$, one has by (21)

$$
\operatorname{Im} \operatorname{tr} \beta(A)=\frac{1}{2 i} \operatorname{tr}\left(\beta(A)-\beta(A)^{*}\right)=\frac{1}{2 i} \operatorname{tr} \beta\left(A-{ }^{t} A\right)=0,
$$

i.e., $\beta(A) \in \mathrm{gl}^{0}(V)$. The implication $\left(\mathrm{ii}_{1}\right)$ or $\left(\mathrm{ii}_{3}\right) \Rightarrow\left(\mathrm{ii}_{4}\right)$ is trivial. Hence it remains to show that $\left(\mathrm{ii}_{4}\right)$ implies one of the conditions $\left(\mathrm{ii}_{1}\right) \sim\left(\mathrm{ii}_{3}\right)$. In [11c], Takeuchi proved $\left(\mathrm{ii}_{4}\right) \Rightarrow\left(\mathrm{ii}_{1}\right)$. Here we give an alternate proof, showing $\left(\mathrm{ii}_{4}\right) \Rightarrow\left(\mathrm{ii}_{3}\right)$. Let $G_{0}$ be the subgroup of $G(\Omega) \times G L(V)$ formed of all pairs $(A, B)$ satisfying the condition

$$
A F\left(v_{1}, v_{2}\right)=F\left(B v_{1}, B v_{2}\right) \quad\left(v_{1}, v_{2} \in V\right) .
$$

Then $G_{0}$ is a real algebraic group with Lie algebra isomorphic to $g_{0}$. The condition $\left(\mathrm{ii}_{4}\right)$ implies that the projection map $p:(A, B) \mapsto A$ gives a homomorphism of $G_{0}^{\circ}$ onto $G(\Omega)^{\circ}$. Since the kernel of $p$ is compact and $G(\Omega)$ is reductive, $G_{0}$ is also reductive. It follows that there exists a Lie algebra homomorphism $\beta: \mathfrak{g}(\Omega) \rightarrow \mathfrak{g l}(V)$ such that $(A, \beta(A)) \in \mathfrak{g}_{0}$ for all $A \in \mathrm{g}(\Omega)$, i.e., $\beta$ satisfies the condition (20). Putting $u=e$ in (20), we see that $A \in \mathfrak{f}_{e}$ implies $\beta(A)=-\beta(A)^{*}$. These mean that the triple $(V$, $\left.\beta \cdot \theta_{e}, f\right)$ is a "solution" for $(\mathfrak{g}(\Omega), e)$ of the problem considered in [10a]. Hence ([10a], p. 127), $\beta$ can be decomposed into a "commutative sum" of two homomorphisms $\beta_{i}(i=0,1)$ of $g(\Omega)$ into $g l(V)$ such that $\beta_{0}(g(\Omega))$ $\subset \mathfrak{u}(V, F)$ and $\beta_{1}$ satisfies the conditions (20) and (21), or in the terminology of [10a], $\left(V, \beta_{1} \cdot \theta_{e}, f\right)$ is a "strong solution" of the same problem. As we noted above, we then have $\beta_{1}(\mathfrak{g}(\Omega)) \subset \mathrm{gl}^{0}(V)$. Thus the condition $\left(\mathrm{ii}_{3}\right)$ is satisfied,

We call a Siegel domain $\mathscr{D}$ quasi-symmetric if the data $(U, V, \Omega, F)$ defining $\mathscr{D}$ satisfy the conditions (i), (ii). Note that this definition does not depend on the expression of $\mathscr{D}$ as a Siegel domain by virtue of its uniqueness. It is clear that a quasi-symmetric Siegel domain is affinely homogeneous. We also remark (after Tsuji [13], Theorem 2.1, and Nakajima) that, if $\mathscr{D}$ is an irreducible quasi-symmetric Siegel domain, 
which is not symmetric, then one has $\mathfrak{g}_{\frac{1}{2}}=\mathfrak{g}_{1}=\{0\}$; by a theorem of Kaup [3] one can then conclude that $\mathrm{Hol}(\mathscr{D})=\operatorname{Aff}(\mathscr{D})$.

By Proposition 1, we have, for a quasi-symmetric Siegel domain $\mathscr{D}$ $=\mathscr{D}(U, V, \Omega, F)$, the following morphisms in three categories:

(a) a strongly equivariant linear map $2 R:(\Omega, e) \rightarrow\left(\mathscr{P}(V, f), 1_{V}\right)$,

(b) a Lie algebra homomorphism $\beta: \mathrm{g}(\Omega) \rightarrow \mathrm{gl}^{0}(V)$ satisfying (20), (21) with $R_{u}=\beta\left(T_{u}\right)$,

(c) a (unital) Jordan algebra homomorphism $2 R: U \rightarrow \mathscr{H}(V, f)$.

For brevity, we call such a morphism (in any of the three categories) a representation on $V$. It is clear that, conversely, given a self-dual cone $(\Omega, e)$ in $U$ and a "representation" $2 R$ of it (or a representation $\beta$ of $\mathrm{g}(\Omega)$ ) on $V$, then defining an $\Omega$-hermitian form $F$ on $V$ by (16), (17), we obtain data $(U, V, \Omega, F)$ satisfying the conditions (i), (ii). Thus a quasisymmetric Siegel domain $\mathscr{D}$ is determined by a pair formed of a selfdual cone $(\Omega, e)$ in $U$ and a "representation" of it on $V .{ }^{7)}$ In particular, $\mathscr{D}$ is a tube domain (Siegel domain of the first kind) $U+i \Omega$, if and only if the corresponding representation is trivial, i.e., $V=\{0\}$. In that case, the condition (iii) being trivially satisfied, $\mathscr{D}$ is necessarily symmetric.

Now, let $\mathscr{D}^{\prime}=\mathscr{D}\left(U^{\prime}, V^{\prime}, \Omega^{\prime}, F^{\prime}\right)$ be another quasi-symmetric Siegel domain determined by a self-dual cone $\left(\Omega^{\prime}, e^{\prime}\right)$ in $U^{\prime}$ and a representation $2 R^{\prime}$ (or $\beta^{\prime}$ ) on $V^{\prime}$. We look for the conditions on these data under which $\mathscr{D}$ and $\mathscr{D}^{\prime}$ are holomorphically equivalent. As is known ([4], Theorem 11), this is the case if and only if $\mathscr{D}$ and $\mathscr{D}^{\prime}$ are linearly equivalent, i.e., there exist a pair of linear isomorphisms

$$
\begin{aligned}
& \varphi: U \longrightarrow U^{\prime}, \\
& \psi: V \longrightarrow V^{\prime}
\end{aligned}
$$

such that

$$
\begin{gathered}
\varphi(\Omega)=\Omega^{\prime}, \\
\varphi\left(F\left(v_{1}, v_{2}\right)\right)=F^{\prime}\left(\psi\left(v_{1}\right), \psi\left(v_{2}\right)\right) \quad\left(v_{1}, v_{2} \in V\right) .
\end{gathered}
$$

Hence, for our purpose, we may assume from the beginning that $U=U^{\prime}$, $\Omega=\Omega^{\prime}$, and, since $\mathscr{D}^{\prime}$ is affinely homogeneous, $e=\varphi(e)=e^{\prime}$ as well. Then one has $\varphi \in G(\Omega), \varphi(e)=e$, i.e., $\varphi \in K_{e}$, which implies ${ }^{t} \varphi=\varphi^{-1}$. The condition (23) is equivalent to

7) The usefulness of the notion of "representations" for Siegel domains was already pointed out by Rothaus [9a]. 


$$
F_{t_{\varphi\left(u^{\prime}\right)}}=F_{u^{\prime}}^{\prime} \cdot(\psi \times \psi) \quad\left(u^{\prime} \in U\right)
$$

which in turn is equivalent to

$$
\begin{array}{cc}
f=f^{\prime} \cdot(\psi \times \psi), & \text { and } \\
R_{u}=\psi^{-1} \cdot R_{\varphi(u)}^{\prime} \cdot \psi \quad(u \in U),
\end{array}
$$

where $f^{\prime}=F_{e}^{\prime}$. These mean that we have the following commutative diagram of Jordan algebra homomorphisms :

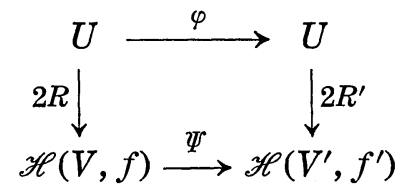

where we put $\Psi(H)=\psi \cdot H \cdot \psi^{-1}$ for $H \in \mathscr{H}(V, f)$. In terms of the corresponding Lie algebra representations $\beta$ and $\beta^{\prime}$, the condition (25) is equivalent to

$$
\beta(A)=\psi^{-1} \cdot \beta^{\prime}\left(\varphi A \varphi^{-1}\right) \cdot \psi \quad(A \in g(\Omega)),
$$

because in view of the relations $\beta\left(T_{u}\right)=R_{u}, \beta^{\prime}\left(T_{u^{\prime}}\right)=R_{u^{\prime}}^{\prime}, \varphi T_{u} \varphi^{-1}=T_{\varphi(u)}$ (25) is equivalent to (26) with $A=T_{u}$ and $g(\Omega)$ is generated by $\mathfrak{p}_{e}$. When the relations (24) and (25) (or (26)) are satisfied for some $\varphi \in K_{e}$ and a linear isomorphism $\psi: V \rightarrow V^{\prime}$, we say two representations $2 R$ and $2 R^{\prime}$ (or $\beta$ and $\beta^{\prime}$ ) are automorphically equivalent (at $e$ ) and write $2 R \approx 2 R^{\prime}$ (or $\beta$ $\approx \beta^{\prime}$ ). Note that the condition (26) alone is sufficient for $\beta \approx \beta^{\prime}$, since we can then modify $\psi$ to satisfy (24) ([10a], Theorem 2). It is clear that, conversely, if $2 R \approx 2 R^{\prime}$ or $\beta \approx \beta^{\prime}$, then the quasi-symmetric Siegel domains $\mathscr{D}$ and $\mathscr{D}^{\prime}$ are linearly equivalent with $\varphi \in K_{e}$. We have thus proved the following

Proposition 2. Two quasi-symmetric Siegel domains $\mathscr{D}$ and $\mathscr{D}^{\prime}$ with the same self-dual cone $(\Omega, e)$ are holomorphically equivalent, if and only if the two "representations" $2 R$ and $2 R^{\prime}$ (or $\beta$ and $\beta^{\prime}$ ) defining $\mathscr{D}$ and $\mathscr{D}$ ' are automorphically equivalent at $e$.

Thus the classification of quasi-symmetric Siegel domains $\mathscr{D}$ with a fixed self-dual cone $(\Omega, e)$ (up to holomorphic equivalence) is equivalent to that of the "representations" $2 R$ or $\beta$ up to automorphic equivalence 
at $e$. The determination of all (non-trivial) "representations" $\beta$ of $g(\Omega)$ was given in [10a]. It was shown there that every such representation is completely reducible and the classification can be reduced to the case where both $\Omega$ and $\beta$ are irreducible. ${ }^{8)}$ For an irreducible self-dual cone $(\Omega, e)$, let $N$ be the number of (ordinary) equivalence classes of (nontrivial) irreducible "representations" $\beta$ of $g(\Omega)$. Then $N \leqq 2$. More precisely, one has $N=2$ for $\Omega=\mathscr{P}_{m}(C)(m \geqq 2)$ and for the "quadratic cones" of even dimension; $N=0$ for the exceptional cone $\mathscr{P}_{3}(\boldsymbol{O})$; and $N$ $=1$ for all other cases. For each of the cases with $N=2$, it is easy to see that two inequivalent irreducible "representations" are automorphically equivalent. In this way, we can reproduce the main result of Takeuchi [11c]. For instance, for $\Omega=\mathscr{P}_{m}(C)(m \geqq 2)$, we define a representation $\beta_{r, s}$ of $\mathfrak{g}(\Omega)=\mathfrak{g l}^{0}(m, C)$ on $V=C^{m(r+s)}$ by

$$
\beta_{r, s}=\overbrace{i d \oplus \cdots \oplus i d \oplus}^{r} \overbrace{i d \oplus \cdots \oplus \overline{i d}}^{s},
$$

$i d$ denoting the identical representation and $\overline{i d}$ its complex conjugate. Then every "representation" $\beta$ of $g(\Omega)$ is automorphically equivalent to one of $\beta_{r, s}(r \geqq s \geqq 0)$. The corresponding quasi-symmetric Siegel domain $\mathscr{D}$ is symmetric if and only if $s=0$, and in that case $\mathscr{D}$ is of type $\left(I_{m+r, m}\right)$.

\section{BIBLIOGRAPHY}

[1] Braun, H. and Koecher, M., Jordan-Algebren, Springer, Berlin-Heidelberg-New York, 1966.

[2] Kaneyuki, S., On the automorphism groups of homogeneous bounded domains, J. Fac. Sci. Univ. Tokyo 14 (1967), 89-130.

[ 3 ] Kaup, W., Einige Bemerkungen über polynomiale Vektorfelder, Jordanalgebren und die Automorphismen von Siegelschen Gebieten, Math. Ann. 204 (1973), 131144.

[4] Kaup, W., Matsushima, Y. and Ochiai, T., On the automorphisms and equivalences of generalized Siegel domains, Amer. J. Math. 92 (1970), 475-498.

[5] Koecher, M., (a) Jordan algebras and their applications, Lecture Notes, Univ. of Minnesota, 1962.

(b) Imbedding of Jordan algebras into Lie algebras, I., Amer. J. Math. 89 (1967), 787-816; II, ibid. 90 (1968), 476-510.

(c) An elementary approach to bounded symmetric domains, Lecture Notes, Rice Univ., Houston, 1969.

8) This may be regarded as a special case of the general decomposition theorem of Siegel domains ([2], [7]).

9) The content of this note is to be incorporated in an author's book on symmetric domains now in preparation. 
[6 ] Murakami, S., On automorphisms of Siegel domains, Lecture Notes in Math., No. 286, Springer, Berlin-Heidelberg-New York, 1972.

[7] Nakajima, K., Some studies on Siegel domains, J. Math. Soc. Japan 27 (1975), 54-75.

[8] Pjateckii-Šapiro, I. I., Geometry of classical domains and theory of automorphic functions (in Russian), Fizmatgiz, Moscow, 1961; English transl., Gordon and Breach, New York, 1969; French transl., Dunod, Paris, 1966.

[ 9 ] Rothaus, O. S., (a) The construction of homogeneous convex cones, Ann. of Math. 83 (1966), 358-376.

(b) Automorphisms of Siegel domains, Trans. Amer. Math. Soc. 162 (1971), 351-382.

[10] Satake, I., (a) Linear imbeddings of self-dual homogeneous cones, Nagoya Math. J. 46 (1972), 121-145; Corrections, ibid. 60 (1976), 219.

(b) Infinitesimal automorphisms of symmetric Siegel domains (Unpublished note, 1974) $^{\text {)). }}$.

[11] Takeuchi, M., (a) On infinitesimal affine automorphisms of Siegel domains, Proc. Japan Acad. 45 (1969), 590-594.

(b) Homogeneous Siegel domains, Publ. Study Group Geometry, No. 7, Tokyo, 1973.

(c) On symmetric Siegel domains, Nagoya Math. J. 59 (1975), 9-44.

[12] Tanaka, N., On infinitesimal automorphisms of Siegel domains, J. Math. Soc. Japan 22 (1970), 180-212.

[13] Tsuji, T., Siegel domains over self-dual cones and their automorphisms, Nagoya Math. J. 55 (1974), 33-80.

[14] Vinberg, E. B., (a) Homogeneous cones, Dokl. Akad. Nauk SSSR 133 (1960), 9-12; = Soviet Math. Dokl. 1 (1960), 787-790.

(b) The theory of convex homogeneous cones, Trudy Moskow Mat. Obšč. 12 (1963), 303-358; = Trans. Moscow Math. Soc. (1963), 340-403.

[15] Vinberg, E. B., Gindikin, S. G. and Pjateckǐ̈-Šapiro, I. I., Classification and canonical realization of complex bounded homogeneous domains, Trudy Moskow Mat. Obšč. 12 (1963), 359-388; = Trans. Moscow Math. Soc. (1963), 404-437.

[16] Wolf, J. and Korányi, A., Generalized Cayley transformations of bounded symmetric domains, Amer. J. Math. 87 (1965), 899-939.

Department of Mathematics

University of California at Berkeley 